\title{
COMPUTATIONAL STUDIES ON THE COGNATE AND NON-COGNATE AMINOACYL-tRNAs
}

\author{
Hadieh Monajemi ${ }^{1}$, Sharifuddin Mohd. Zain ${ }^{2}$, Wan Ahmad Tajuddin Wan \\ Abdullah ${ }^{1,3}$ \\ ${ }^{1}$ Department of Physics, Universiti Malaya, Kuala Lumpur, Malaysia, ${ }^{2}$ Department of Chemistry, \\ Universiti Malaya, Kuala Lumpur, Malaysia, ${ }^{3}$ Faculty of Computer Science and Information \\ Technology, Universiti Malaya, Kuala Lumpur, Malaysia \\ *h.monajemi@hotmail.com (Corresponding author) \\ Received on $3^{\text {rd }}$ May 2011 , accepted in revised form $20^{\text {th }}$ July 2011
}

\begin{abstract}
Although kinetic proofreading plays a major role in the fidelity of protein synthesis, it only includes the codon-anticodon interaction without considering the amino acid side chain. It is not known whether the ribosome has a specific interaction with the amino acid side chain or if the correctly charged tRNA behaves in such a way that brings this fidelity to protein synthesis process. To answer this question, we performed some preliminary calculations using numerical analysis on the misacylated tRNAs and compared the results with that of the cognate aminoacyltRNAs. Due to the large size of the molecule, we used 2-layered ONIOM (QM/MM) calculations. Interestingly, it was observed that only the initiator $\operatorname{tRNA}_{i}{ }^{\text {met }}$ has a specific interaction with the amino acid side chain.
\end{abstract}

(Keywords: Protein synthesis, aminoacyl-tRNA, ribosome.)

\section{INTRODUCTION}

Ribosome is a large macromolecular complex that translates the genetic code from mRNA (messenger ribonucleic acid) to proteins. It is made of two subunits, i.e. the smaller subunit and the larger subunit. The mRNA molecule binds to the smaller subunit and the peptide bond between the amino acids is formed in the larger subunit. The tRNA (transfer ribonucleic acid) molecule which is in charge of transferring the amino acids to the ribosome binds from anticodon end to the mRNA codon in the smaller subunit and from amino acid end to the PTC (peptidyl transferase center) in the larger subunit ${ }^{1}$. The tRNA aminoacylation occurs in another enzyme called aminoacyltRNA synthetase (aaRS). The error rate is on the order of 1 in $10^{4}$ for this process ${ }^{2}$. The proper arrangement of amino acids is very important for the proteins' structure. This arrangement is based on the arrangement of codons on the mRNA molecule. It is vital to have a high fidelity in codon-anticodon interaction in order to have a proper arrangement of amino acids. This fidelity is achieved by the kinetic proofreading process in which the discrimination between the two cognate and near/non-cognate (in terms of their anticodon interaction with the mRNA codon) aminoacyl-tRNAs is based on the rate of their accommodation in the ribosome. The lower rate of accommodation for the near/non-cognate aminoacyl-tRNA structures results in their rejection from the ribosome ${ }^{3}$. However, the amino acid attached to the tRNA is not involved in the kinetic proofreading process. On the other hand, it is not clear whether the ribosome specifies the amino acid side chain during initial selection since the incorporation rate of misacylated tRNAs compared to the correctly acylated ones, is significantly similar ${ }^{4}$. Therefore, a misacylated tRNA can be a serious threat to the fidelity of protein synthesis unless either the tRNA specifies its bound amino acid or the ribosome specifies the misacylated tRNA. However, it is not known at which stage of elongation the misacylated tRNA goes wrong. The results reported in this paper 
illustrate the structural behavior of misacylated and correctly acylated tRNA molecules in the absence of ribosomal bases. This study is carried out to investigate the first assumption as aforementioned.

\section{COMPUTATIONAL METHODS}

The X-ray crystal structures of five different tRNAs were taken from the Protein Data Bank (ID codes: 1YFG, 1F7V, 1G59, 1QRS, and 1EVV for , tRNA $^{\text {met }}$, tRNA ${ }^{\text {arg }}$, tRNA $^{\text {glu }}$, tRNA $^{\text {gln }}$, and tRNA ${ }^{\text {phe }}$ respectively) ${ }^{16,17,18,19,20}$ and were used as the starting geometries of all computations. Due to the large tRNA molecular size, a 2-layer ONIOM (QM/MM) (Our own Nlayered Integrated molecular Orbital and molecular Mechanics) method ${ }^{11,15}$ is used in order to have an accurate and computationally feasible calculation. The amino acid arm of the tRNA (QM region) is treated with MP2 method while the rest of the tRNA (MM region) is treated with UFF method (refer to Figure 1). The boundary region is treated with the link atom model and the electrostatic interaction between the two regions is handled with Electronic Embedding (EE) where the partial charges from MM region are included in the QM Hamiltonian. This brings more accuracy to the calculations compared to the Mechanical Embedding (ME) which is used in Gaussian by default. We used doublet multiplicity for the real system and singlet multiplicity for both low and high level of calculation on the model system.

Five different amino acids (i.e. methionine (met), arginine (arg), glutamic acid (glu), glutamine (gln), and phenylalanine (phe)) were selected based on the availability of their corresponding tRNA's crystal structure in the database. These amino acids were attached to each of the five different tRNAs. This results in 25 different aminoacyl-tRNAs of which 5 are cognate and 20 are non-cognate structures. We have applied different conditions to the molecule by different substitutions on the amino acid moiety, i.e. the halogen atom substitution is performed where the hydrogen of the alpha carbon $(\mathrm{C} \alpha)$ is substituted with four different halogen atoms, and methyl (electron donating), ethyl (electron donating), and phenyl (electron withdrawing) substitutions were made wherein the hydrogen atom attached to the amino nitrogen is substituted with the methyl, ethyl and phenyl groups ${ }^{12}$. Only SPE (single point energy) calculations using ONIOM(MP2/6-31G (d,p):UFF) is performed on the molecule. The geometry of the amino acid molecule on the other hand, is optimized before its attachment to the tRNA is done at the MP2 level. All geometry optimizations and single point calculations were carried out at $298.15 \mathrm{~K}$ and 1 atm pressure. All computations were carried out using the Gaussian 09 program $^{10}$. Molecular visualization was facilitated with the use of Gauss View ${ }^{10}$. The calculations were performed on the Intel Pentium D PC Desktop, running windows XP, using only one processor.

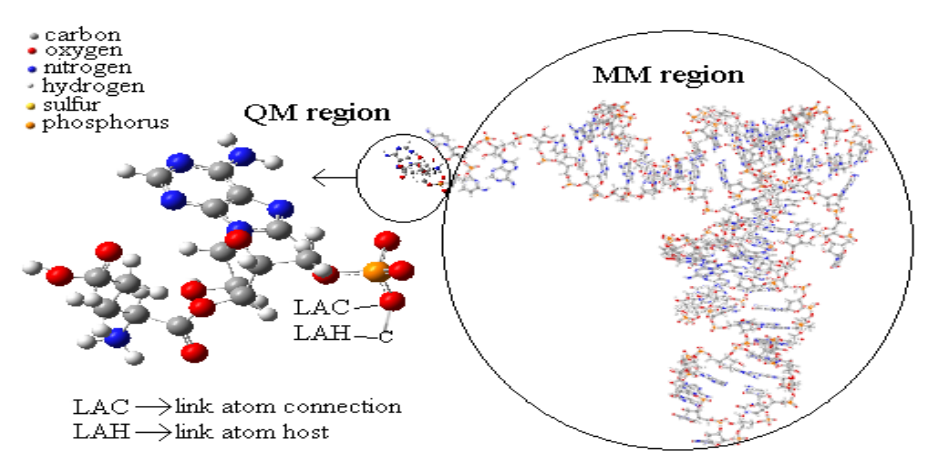

Figure 1. ONIOM partitioning model: QM region is where the peptide bond formation occurs 


\section{RESULTS AND DISCUSSION}

The relative energy variation (REV) with respect to the increase in electronegativity for different aminoacyl-tRNA molecules is shown in Figure 2 below. The main graph shows the energy variation for the initiator $\mathrm{tRNA}_{\mathrm{i}}{ }^{\text {met }}$ attached to the five amino acids. The four smaller graphs inset show the energy variation for the other four elongator tRNA structures attached to the same amino acids. The second vertical axis on the right hand side of the main graph belongs to the relative energy of the cognate met-tRNA ${ }_{i}^{\text {met }}$, which is too large in scale to show similarly in the scale with the non-cognate ones (i.e. most severe for iodine substituent).

The lowest ionization energy on the molecule is caused by iodine substituent which results in a relatively unstable structure. Moreover, the high polar ester bond caused by iodine substitution makes the structure more likely to undergo chemical reactions. This only affects mettRNA $_{i}{ }^{\text {met }}$ and the other aminoacyl-tRNAs show similar behavior in different substitutions. Furthermore, the met-tRNA ${ }_{i}{ }^{\text {met }}$ molecule has high stability compared to the misacylated tRNA $_{i}{ }^{\text {met }}$ structures as well as to cognate and non-cognate elongator aminoacyl-tRNAs in the normal condition with the presence of the hydrogen atom. Since the ester bond is highly labile $^{13}$, the high stability of the met-tRNA ${ }_{i}^{\text {met }}$ under normal unsubstituted condition prevents the cognate methionine from being hydrolyzed. This stability is not observed for the cognate elongator aminoacyl-tRNAs which indicates that there is a difference between the structure of initiator and elongator tRNAs and the way they function under different conditions. It is worth mentioning that due to the high electronegativity of fluorine; the system breaks down under fluorine atomic substitution and shows the lowest energy possible for all tRNAs which is not reasonable. This illustrates the high polarizability of the aminoacyl-tRNA structure.

To further investigate the structural behavior of met-tRNA ${ }_{i}{ }^{\text {met }}$ and slightly change the external filed applied on the molecule, we have studied the effects of substitution on the relative energy of the cognate and non-cognate aminoacyl-tRNAs by substituting electron donating groups (EDG) and electron withdrawing groups (EWG) on the hydrogen atoms attached to the amino nitrogen of the amino acid moiety. Methyl, ethyl, and phenyl groups were used as substituents on one and both hydrogen atoms of the amino nitrogen. Based on the atom which these groups are attached to (i.e. N), each of these groups act as either EDG or EWG. That is, the methyl and ethyl groups have an electron donating inductive effect $(+\mathrm{I})$ on the amino acid moiety while the phenyl group has an electron withdrawing mesomeric effect (-M). As observed in Figure 3 and Figure 4 below, the REV is almost similar for elongator tRNAs. On the other hand, we found the initiator $\mathrm{RNA}_{\mathrm{i}}{ }^{\mathrm{met}}$ behavior to be different when attached to noncognate amino acids. In contrast to elongator tRNAs, met-tRNA ${ }_{i}^{\text {met }}$ demonstrates a high stability in the normal condition with no substitution, which is imperative in order to protect the methionine from being hydrolyzed when met-tRNA ${ }_{i}{ }^{\text {met }}$ is attached to the complex of mRNA-smaller ribosomal subunit before attachment to the larger ribosomal subunit. During the elongation process, this role is played by the universally conserved rRNA base U2585 which protects the peptidyl tRNA's ester bond from being hydrolyzed when the Asite is empty ${ }^{14}$ (after translocation), and the initiator met-tRNA ${ }_{i}^{\text {met }}$ lacks this protection when it is not yet bound to the larger ribosomal subunit. Such behavior is similar to that in electronegative atomic substitutions. The unsubstituted amino acid structure is most stable for cognate met-tRNA ${ }_{i}{ }^{\text {met }}$ compared to the other structures (refer to Figure 2).

The arginine amino acid on the other hand, with a basic side chain and positive charge, is expected to attract electrons from the active site (i.e. the carboxyl group attached to the ribose) which results in a more stable molecule. Moreover, the presence of EDG in the amino side of arginine results in positive charge at the other end of arginine moiety (i.e. the carboxyl group). However, the behavior of this amino acid happens to be different when attached to the $\mathrm{tRNA}_{\mathrm{i}}{ }^{\mathrm{met}}$. $\mathrm{Arg}-\mathrm{tRNA}_{\mathrm{i}}{ }^{\mathrm{met}}$ is relatively unstable in the presence of EDG and under normal unsubstituted condition (Figure 4). This would ultimately lead to the hydrolysis of the structure and dissociation of the non-cognate amino acid from the initiator $\mathrm{tRNA}_{\mathrm{i}}{ }^{\mathrm{met}}$. The situation is almost the same for $g \ln -\mathrm{RNA}_{\mathrm{i}}{ }^{\text {met }}$ and phe-tRNA $\mathrm{A}_{\mathrm{i}}{ }^{\mathrm{met}}$. These different behaviors of tRNA $_{i}{ }^{\text {met }}$ for different amino acids compared to 
the elongator tRNAs indicate the importance of the amino acid side chain identity for the initiator tRNA ${ }_{i}{ }^{m e t}$. Knowing that the advantage of elongator tRNAs over $\operatorname{tRNA}_{i}{ }^{\text {met }}$ is the former's interaction with the ribosomal A-site, the results shown in this study demonstrate the importance of the larger ribosome in stabilizing the correct acylated tRNA at the A-site. Furthermore, the initiator $\operatorname{tRNA}_{i}{ }^{\text {met }}$ binds initially to the P-site of the larger ribosome, the site which is less likely to bind to the amino acid side chain compared to the A-site. Therefore, the difference between initiator tRNA $_{i}{ }^{\text {met }}$ and other elongator tRNAs is in the latter's tendency to be stabilized at the ribosomal A-site in its interaction with the ribosome, and in the former's less likeliness to be stabilized because of its initial attachment to the P-site. This indicates the lack of substrate specification at the P-site which agrees well with existing biochemical data ${ }^{14}$.

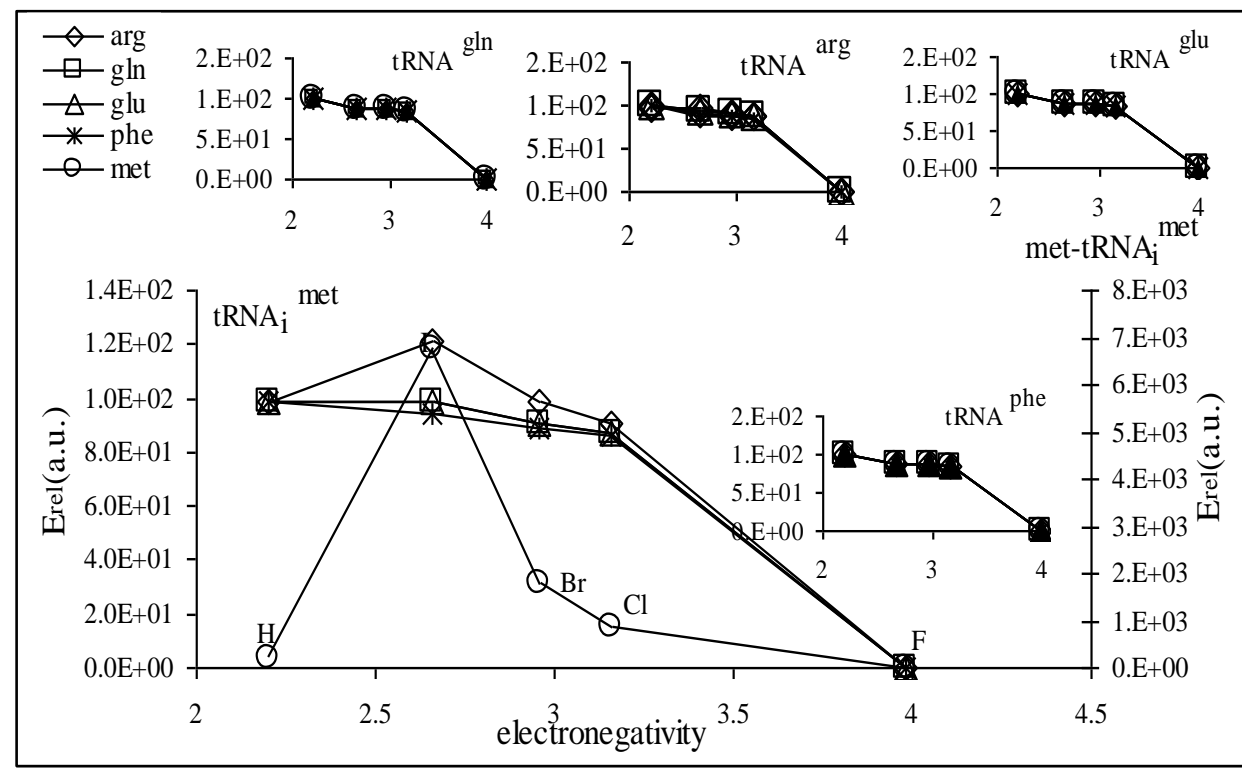

Figure 2. The REV for cognate and non-cognate aminoacyl-tRNAs with electronegative substitutions.

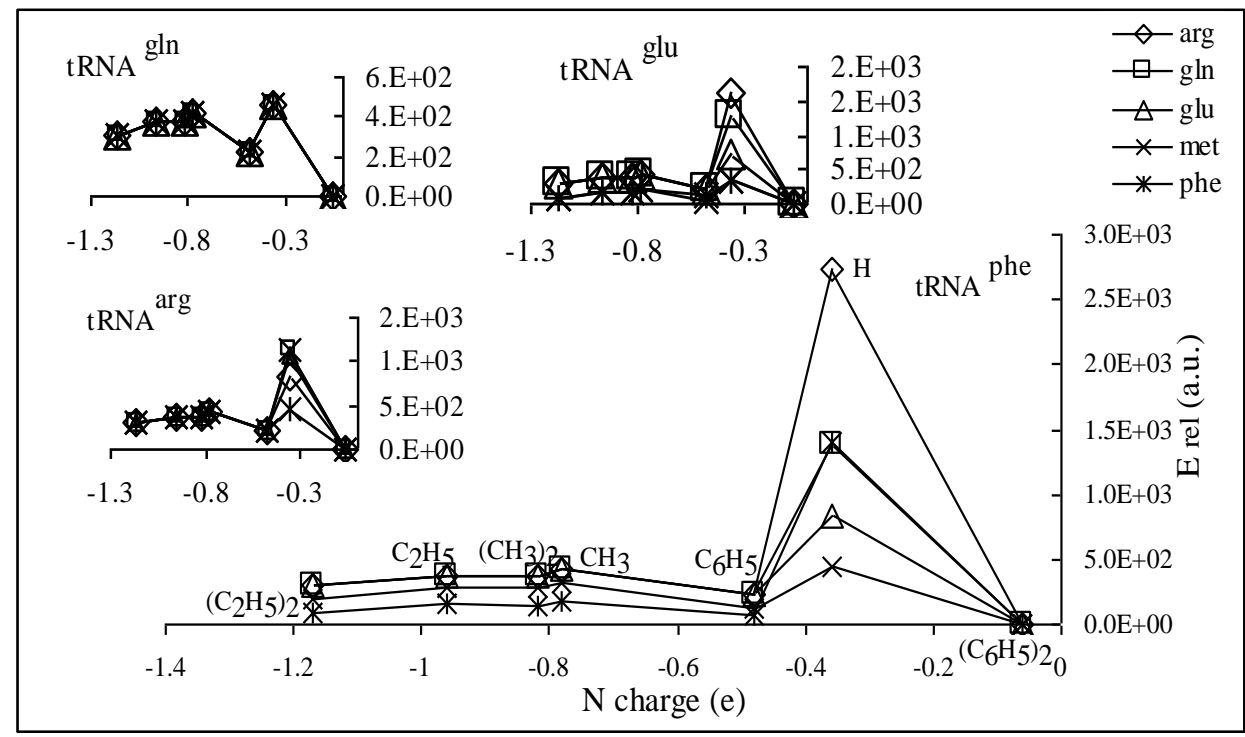

Figure 3. The REV for cognate and non-cognate elongator aminoacyl-tRNAs with EDG and EWG substitutions. 


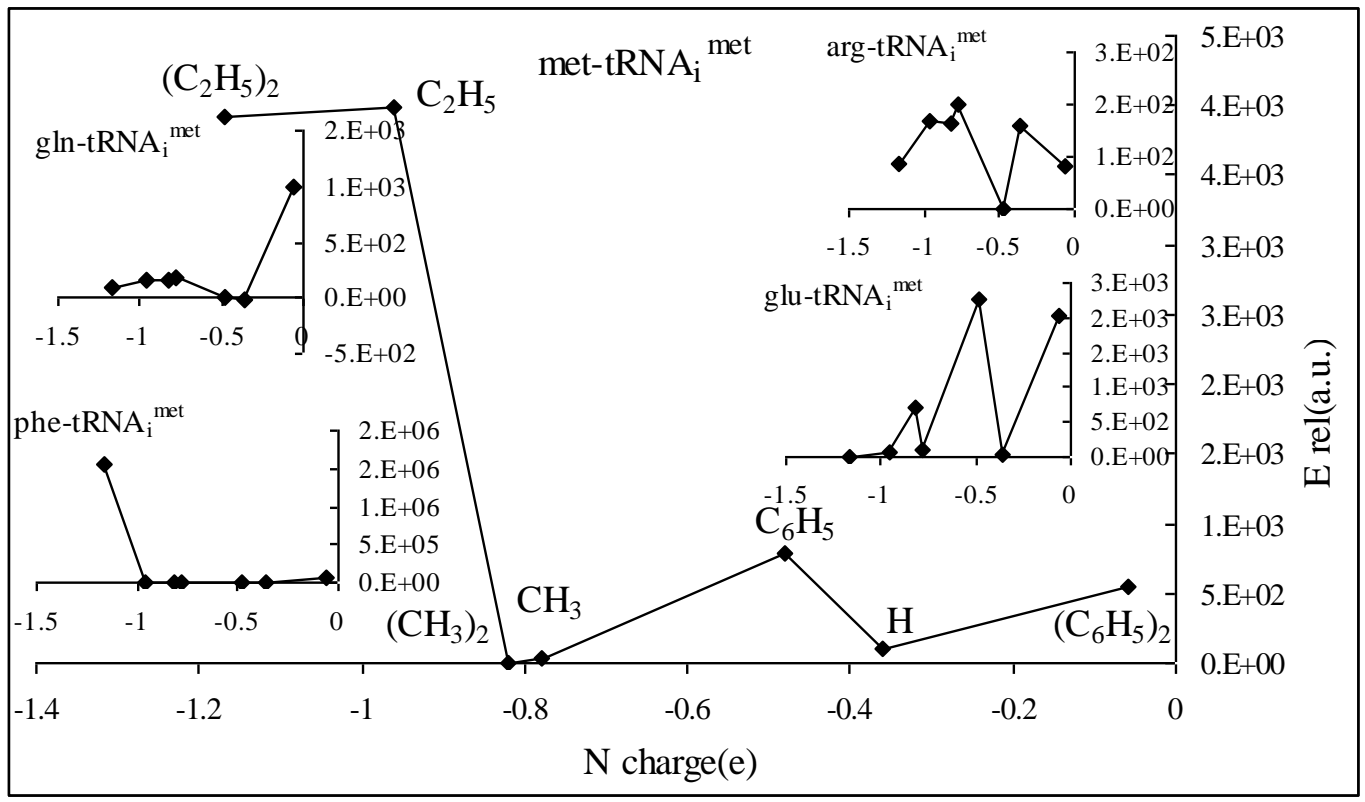

Figure 4. The REV for cognate and non-cognate initiator aminoacyl-tRNA ${ }_{i}^{\text {met }}$ with EDG and EWG substitutions.

\section{REFERENCES}

1. T. Martin Schmeing and V. Ramakrishnan, Nature 461, 12341242 (2009).

2. Stortchevoi, Alexei. A., Cellular and Molecular Life Science $\mathbf{6 3}$, 820-831 (2006).

3. L. Cochella, and R. Green., Current Biology 15, 536-540 (2005).

4. P.R. Effraim, J. Wang, M.T. Englander, J. Avins, T.S. Leyh, R.L. Gonzalez Jr, and V.W. Cornish., Nature Chemical Biology 5, 947-953 (2009).

5. Basavappa, R., Sigler, P.B, EMBO J. 10, 3105-3111 (1991).

6. Delagoutte, B., Moras, D., Cavarelli, J., EMBO J. 19, 55995610 (2000).

7. Sekine, S., Nureki, O., Shimada, A., Vassylyev, D.G., Yokoyama,
8. S., Nature Structural \& molecular Biology 8, 203-206 (2001).

9. Arnez, J.G., Steitz, T.A., Biochemistry 35, 14725-14733 (1996).

10. Jovine, L., Djordjevic, S., Rhodes, D., J.Mol.Biol. 301, 401414 (2000).

11. Gaussian 09, J.A.Pople, Gaussian, Inc., Pittsburgh PA, 2009.

12. Vreven, Thom., Byun, K. Suzie,, Komromi, Istvn., Dapprich, Stefan., Montgomery, John. A., Morokuma, Keiji., and Frisch, Michael. J., Journal of Chemical Theory and Computation 2, 815826 (2006).

13. Pavlov, M. Y., Whatts, R. E., Tan, Z., Ehrenberg, V. W. C. M. \& Forster, A. C. Proceedings of the National Academy of Sciences of the United States of America 106, 50-54 (2009).

14. Stepanov, Victor G., and Nyborg, Jens., Extremophiles 6, 485-490 (2002). 
15. Voorhees, Rebecca. M., Weixlbaumer, Albert., Loakes, David., Kelley, Ann C., and Ramakrishnan, V., Nature Structural \& Molecular Biology 16, 528-533 (2009).

16. Morokuma, K., Bulletin of the Korean Chemical Society $\mathbf{2 4}$, 797-801 (2003).

17. Basavappa, R., Sigler, P.B., EMBO J. 10, 3105-3111 (1991).

18. Delagoutte, B., Moras, D., Cavarelli, J., EMBO J. 19, 55995610 (2000).

19. Sekine, S., Nureki, O., Shimada, A., Vassylyev, D.G., Yokoyama, S., Nature Structural \& molecular Biology, 8, 203-206 (2001).

20. Arnez, J.G., Steitz, T.A., Biochemistry 35, 1472514733(1996).

21. Jovine, L., Djordjevic, S., Rhodes, D., J.Mol.Biol. 301, 401414 (2000). 